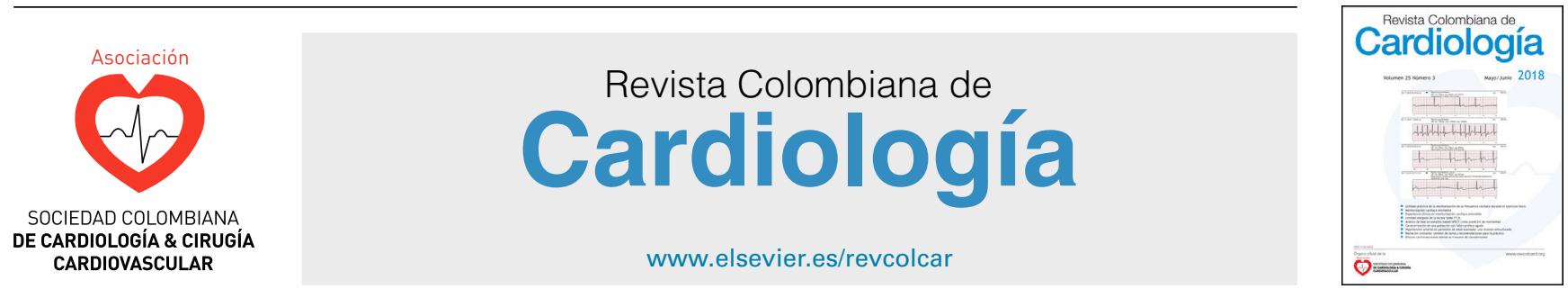

CARDIOLOGÍA PEDIÁTRICA - REVISIÓN DE TEMAS

\title{
Falla cardíaca en pacientes pediátricos. Fisiopatología y tratamiento. Parte II
}

\section{Gabriel Cassalett-Bustillo}

\author{
UCI pediátrica, Fundación Clínica Shaio, Bogotá, Colombia
}

Recibido el 22 de marzo de 2017; aceptado el 7 de marzo de 2018

Disponible en Internet el 28 de junio de 2018

\section{PALABRAS CLAVE \\ Falla cardiaca; \\ Agentes inotrópicos; \\ BNP; \\ Miocardiopatía}

\section{KEYWORDS}

Heart failure;

Inotropic agents

\begin{abstract}
Resumen La falla cardiaca en una patología poco reconocida en la edad pediátrica y tiene una alta tasa de mortalidad al no ser diagnosticada en forma temprana. Se hace una revisión del diagnóstico, la estratificación y el manejo actual de la falla cardiaca en niños y se mencionan las nuevas terapias actualmente en investigación.

(C) 2018 Sociedad Colombiana de Cardiología y Cirugía Cardiovascular. Publicado por Elsevier España, S.L.U. Este es un artículo Open Access bajo la licencia CC BY-NC-ND (http:// creativecommons.org/licenses/by-nc-nd/4.0/).
\end{abstract}

\section{Heart failure in paediatric patients: pathophysiology and treatment. Part II}

\begin{abstract}
Heart failure is a little known condition at paediatric age, and has a high mortality rate on not being diagnosed early. A review is presented on its diagnosis, stratification, and current management of heart failure in children, as well the new therapies currently under investigation.

(c) 2018 Sociedad Colombiana de Cardiología y Cirugía Cardiovascular. Published by Elsevier España, S.L.U. This is an open access article under the CC BY-NC-ND license (http:// creativecommons.org/licenses/by-nc-nd/4.0/).
\end{abstract}

\section{Tratamiento de la falla cardíaca}

La falla cardíaca en pacientes pediátricos es una entidad que conlleva morbilidad y mortalidad importante de no ser diagnosticada y tratada de manera adecuada. Es secundaria a gran número de enfermedades congénitas o adquiridas;

Correo electrónico: gabcass@yahoo.com el manejo puede variar de acuerdo con la etiología y la anatomía cardíaca.

La meta es mejorar los síntomas, evitar la progresión del daño miocárdico y, hasta cierto punto, evitar la remodelación cardíaca, ya que ésta hace parte del desarrollo normal del corazón pediátrico.

El manejo de la falla cardíaca en niños se basa en los estudios de la población adulta. No hay estudios aleatorizados pediátricos con un gran número de pacientes y la mayoría 


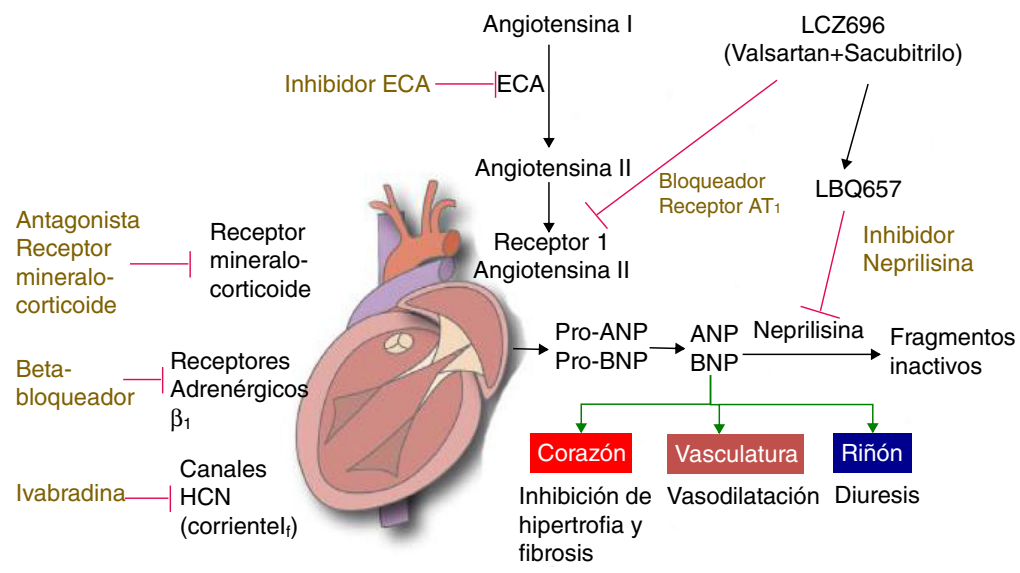

Figura 1 Medicamentos actuales para falla cardíaca Modificado de Lother y Hein².

de las recomendaciones se basa en estudios pequeños, presentación de casos y recomendaciones de expertos.

Para el enfoque del manejo es preciso tener en cuenta la anatomía cardíaca; no es igual cuando hay un ventrículo único derecho, un ventrículo único izquierdo o un corazón biventricular.

Muchos de los medicamentos recomendados en la actualidad para adultos no tienen autorización para uso en niños y aunque han sido suministrados por algunos grupos, no pueden convertirse en una recomendación formal.

En la figura 1 se resumen las recomendaciones del enfoque inicial en el tratamiento de la falla cardíaca; el manejo se instaura de acuerdo con la sintomatología y la respuesta a las terapias ${ }^{1}$.

Para la población adulta hay un sinnúmero de medicamentos que se describen en la figura 1, pero para los niños la disponibilidad es limitada y la evidencia de su empleo es escasa o poco fuerte ${ }^{2}$.

Al ser una enfermedad de fisiopatología multifactorial, el manejo médico se basa en la combinación de varios medicamentos que actúan sobre los diferentes mecanismos involucrados en la génesis o perpetuación de la falla $a^{3,4}$.

A continuación se mencionan los grupos de medicamentos que se pueden emplear ${ }^{5}$ :

1. Diuréticos.

2. Inotrópicos.

3. Nuevos inotrópicos.

4. Inhibidores de la enzima convertidora de angiotensina (IECA), bloqueadores de receptores de la angiotensina II (ARAII).

5. Beta-bloqueadores.

6. Análogos del péptido natriurético.

7. Bloqueadores de los receptores de la vasopresina.

8. Sistemas de soporte extracorpóreo.

9. Terapias génicas.

10. Células madre.

La reanimación con líquidos para el manejo de la falla cardiaca aguda está contraindicada y puede empeorar los síntomas. La figura 2 corresponde a un mapa de ruta de manejo inicial de la falla cardiaca aguda publicado en 2010, sugerido por Kantor y Mertens ${ }^{1}$

\section{Diuréticos}

La mayoría de pacientes pediátricos con falla cardíaca presentan cuadro de falla respiratoria, que puede ser incipiente e inicialmente obedece al cúmulo de líquido en el espacio intersticial. Independiente de la etiología la mayoría de pacientes con falla cardíaca mejora con el empleo de diuréticos (fig. 3).

Además de la disminución del líquido intersticial la reducción de la precarga en un ventrículo funcionando al máximo de la tensión de la fibra puede mejorar la contractilidad ${ }^{6,7}$.

Los diuréticos hacen parte importante del manejo y se deben emplear con precaución. La literatura que demuestra una disminución en la mortalidad con su empleo en la población pediátrica, es muy limitada.

Los diuréticos de asa son los más usados en la presentación aguda de la falla cardíaca.

El más empleado es la furosemida, un diurético de asa que inhibe la reabsorción de electrolitos en la rama ascendente del asa de Henle. La dosis usual de inicio en la falla aguda es de 0,5 a $1 \mathrm{mg} / \mathrm{kg}$ por vía oral o intravenosa. Cuando se usan dosis superiores a $1 \mathrm{mg} / \mathrm{kg} /$ día se recomienda asociar diuréticos ahorradores de potasio.

En adultos el empleo de furosemida a grandes dosis por tiempo prolongado se ha asociado con un incremento en la mortalidad $^{8}$.

En los pacientes desnutridos la furosemida debe administrarse cada 6 u 8 horas para obtener un mejor efecto. Debido a que se liga a proteínas, el no tener niveles de proteínas normales disminuye la vida media del medicamento. En recién nacidos se debe tener cuidado por el riesgo de nefrocalcinosis y nefrolitiasis con su uso prolongado?.

Otros diuréticos que se emplean son las tiazidas, que actúan después del asa de Henle y son útiles cuando la respuesta a la furosemida es limitada.

La espironolactona es un diurético ahorrador de potasio poco potente pero muy utilizado en el manejo de falla cardíaca por sus propiedades ahorradoras de potasio y por su posible efecto de disminución de la fibrosis miocárdica 


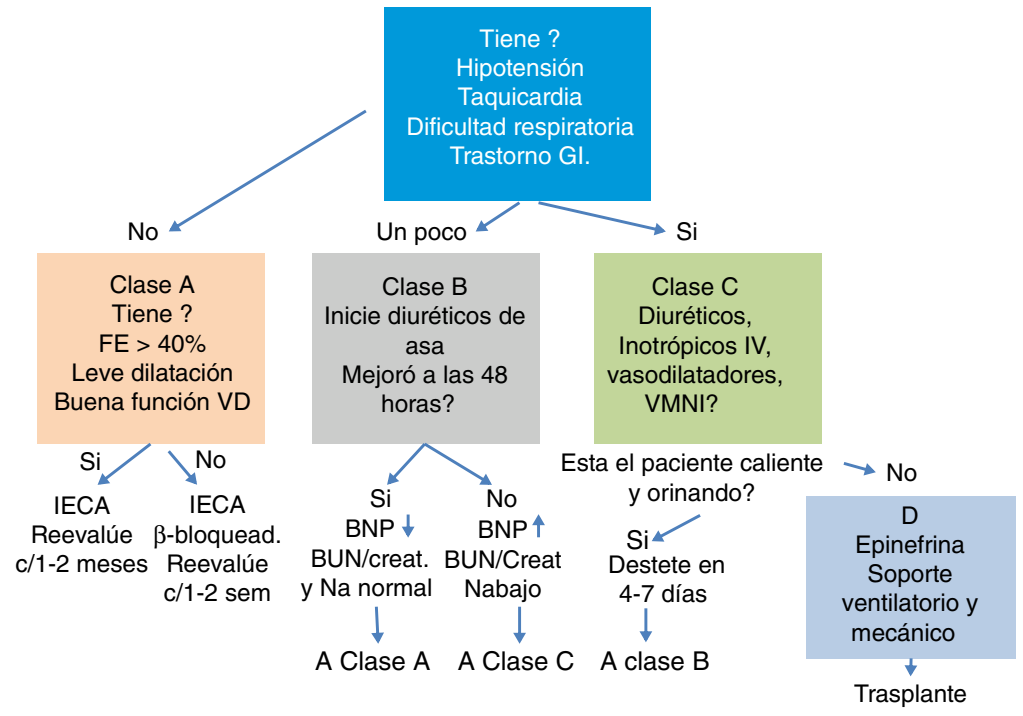

Figura 2 Enfoque de manejo inicial de la falla cardíaca Modificado de Kantor y Mertens ${ }^{1}$.



Figura 3 Hoja de ruta en el manejo de la falla cardíaca. Modificada de Kantor y Mertens ${ }^{1}$.

inducida por niveles altos de aldosterona circulante. Se recomienda su uso en pacientes que reciben IECA y betabloqueadores y no han tenido mejoría de la función o regresión de la remodelación.

Siempre se deben hacer controles de potasio sérico, especialmente cuando se asocia a IECA.

\section{Inotrópicos}

Los medicamentos inotrópicos que se pueden emplear en falla cardíaca pertenecen a varios grupos.

a. Digital.

b. Adrenérgicos.

c. Inhibidores de la fosfodiesterasa III.

d. Sensibilizadores de las proteínas contráctiles por el calcio.

El empleo de inotrópicos está justificado en pacientes que ingresan con cuadro significativo de bajo gasto o shock cardiogénico de cualquier la causa. Para escoger un inotrópico se deben conocer los beneficios que tienen algunos sobre los otros.

En general, el empleo de inotrópicos para el manejo de la falla cardíaca crónica o aguda en adultos es controversial y debería limitarse a falla cardíaca que no responde a diuréticos y vasodilatadores o como tratamiento puente en pacientes en lista de espera para trasplnte $\operatorname{cardíaco}^{10}$.

\section{Digital}

El empleo de este medicamento para el manejo de la falla cardíaca es controvertido, debido a que la mayoría de niños tienen una función sistólica conservada. Sus efectos benéficos además de la inhibición de la bomba sodio-potasio ATPasa que aumenta los niveles de calcio intracelular, son: disminución en los niveles de norepinefrina plasmática, en la actividad del sistema nervioso simpático y de la actividad del sistema renina-angiotensina-aldosterona e incremento del tono vagal y normalización de barorreceptores arteriales.

La digital se puede emplear con precaución en casos agudos con cambios en la perfusión y función renal, también cuando existen cambios rápidos en las concentraciones de potasio y calcio.

Las concentraciones séricas de digoxina pueden ser impredecibles cuando se co-administra con carvedilol llegando potencialmente a efectos tóxicos ${ }^{11}$. Se debe anotar que el empleo de digital en la falla cardíaca crónica no disminuye la mortalidad ${ }^{12}$. En falla crónica deben usarse dosis bajas.

Una de las indicaciones que continúa vigente es la persistencia de la disfunción ventricular izquierda a pesar de un tratamiento médico adecuado.

\section{Adrenérgicos}

En general el empleo de medicamentos que incrementen los niveles de norepinefrina circulante debe ser cauteloso 
puesto que aumentan el consumo de oxígeno miocárdico; por tanto, solo deben administrarse en casos de reagudización de la falla cardíaca con compromiso de la presión arterial y en el menor tiempo posible.

La dopamina aumenta la frecuencia cardíaca, el volumen latido y la resistencia vascular sistémica en forma dosisdependiente.

La adrenalina y la noradrenalina elevan la frecuencia cardíaca, el volumen latido y la resistencia vascular sistémica, pero incrementan aún más el consumo de oxígeno miocárdico y se asocian a la presentación de arritmias.

La dobutamina tiene un efecto vasodilatador sistémico, que aumenta el volumen latido.

\section{Inhibidores de la fosfodiesterasa III}

El único medicamento disponible es la milrinona; su mecanismo de acción se da a través del incremento de los niveles del AMPcíclico (adenosín monofosfato cíclico) intracelular inhibiendo su degradación por la fosfodiesterasa. Su efecto se produce por el incremento en los niveles de calcio intracelular y hay más calcio disponible para el acople actina-miosina.

Está indicado en disfunción diastólica y mejora el lusitropismo y la función ventricular.

La milrinona al no tener efecto adrenérgico, no incrementa la presión pulmonar y está indicada en pacientes con hipertensión pulmonar por su efecto vasodilatador pulmonar moderado. Para tener un buen efecto vasodilatador pulmonar hay que emplear dosis altas de milrinone (mayores de $0,5 \mathrm{mcg} / \mathrm{kg} / \mathrm{min}$ ); muchos pacientes presentan hipotensión sistémica, hecho que limita su uso a dosis altas.

\section{Sensibilizadores de calcio}

Aumentan la afinidad de las proteínas contráctiles por el calcio; no incrementan los niveles de AMP cíclico ni los de norepinefrina. El levosimendan está indicado en falla cardíaca crónica agudizada. Su mayor beneficio se observa en pacientes con miocardiopatía dilatada secundaria a lesiones isquémicas.

Diferentes trabajos han demostrado su utilidad o futilidad comparado con placebo en la prevención del bajo gasto postoperatorio $^{13,14}$.

\section{Nuevos inotrópicos}

Desde hace más de una década no hay nuevos inotrópicos en uso clínico. La búsqueda se ha centrado en mecanismos diferentes al estímulo de los receptores adrenérgicos.

A continuación se enumeran algunos de los nuevos inotrópicos en diferentes fases de desarrollo ${ }^{14,15}$.

\section{Istaroxime}

Su mecanismo de acción es la inhibición de la $\mathrm{Na}^{+}-\mathrm{K}^{+}$-ATPasa y el estímulo del SERCA (Calcio ATPasa del retículo sarcoplasmático). En animales de experimentación se ha demostrado que incrementa la contracción y la relajación. No aumenta el consumo de oxígeno miocárdico. El estudio HORIZON en adultos demostró que incrementó las cifras de tensión arterial y el gasto cardíaco en forma transitoria.

\section{Omenctiv mecarbil}

Activa la formación de puentes actina-miosina. Al incrementar el número de puentes que se forman hay mejoría en la contracción. Los estudios preliminares en humanos demostraron un incremento en la fracción de acortamiento y en la fracción de eyección.

\section{Etomoxir y piruvato}

El Etomoxir es un inhibidor de la palmitoiltransferasa I, que normaliza las bombas de calcio y una porción de la isoenzima V1 de la miosina que se encuentra alterada en los miocardios hipertróficos con sobrecarga de presión y volumen.

El piruvato es un sustrato directo para la producción de energía. Incrementa la actividad contractil de miocitos humanos aislados.

\section{Estabilizador del receptor de rianodina (\$44121)}

Equilibra el receptor de rianodina para modular la salida de calcio durante la diástole. Al disminuir la salida de calcio del retículo sarcoplasmático hay mayor concentración de calcio para la contracción, mejora la relajación y disminuye la presencia de arritmias por exceso de calcio en el citosol durante la diástole.

\section{Donantes de nitroxilo (CXL.1020)}

De estos hay dos medicamentos nuevos el istaroxime y el omencativ. El mecanismo de acción del istaroxime es la inhibición de la $\mathrm{Na+-K+-ATPasa} \mathrm{y} \mathrm{el} \mathrm{estímulo} \mathrm{del} \mathrm{calcio} \mathrm{ATPasa}$ del retículo sarcoplásmico (SERCA).

En animales de experimentación se ha demostrado que incrementa la contracción y la relajación. No incrementa el consumo de oxígeno miocárdico. El estudio HORIZON en adultos demostró que incrementó las cifras de presión arterial e incrementó el gasto cardíaco en forma transitoria.

El omenctiv mecarbil activa la formación de puentes actina-miosina. Al incrementar el número de puentes que se forman hay una mejora contracción. Los estudios preliminares en humanos demostraron un incremento en la fracción de acortamiento y la fracción de eyección.

\section{Inhibidores de la enzima convertidora de angiotensina}

Su uso ha demostrado en adultos en diferentes estudios una disminución importante en la mortalidad ${ }^{16-19}$ y no solo disminución de la mortalidad sino de la morbilidad ${ }^{20,21}$. Inhiben la enzima responsable de la conversión de angiotensina I a angiotensina II, lo que produce una disminución de los niveles plasmáticos de angiotensina II y aldosterona, y causa vasodilatación periférica.

Otro efecto es la reducción del cortocircuito de izquierda a derecha en niños con cardiopatías pero con resistencia 
vascular pulmonar normal; sin embargo, no se encontró ningún efecto sobre el cortocircuito cuando existe hipertensión pulmonar ${ }^{22}$.

A diferencia de los adultos no hay ningún estudio que avale el empleo rutinario de enalapril en pacientes pediátricos con falla cardíaca asintomáticos.

Un trabajo reportó que el empleo de enalaprilat por cortos períodos de tiempo en fisiología de ventrículo único en pacientes en postoperatorio de Glenn con función normal, produjo redistribución del flujo hacia la parte inferior del cuerpo y causó desaturación arterial ${ }^{23}$.

En el grupo de los IECA hay varios medicamentos que han sido empleados en niños además de enalapril y la de captopril. Estos son el lisinopril y el ramipril, aunque solo hay pequeños reportes de su uso en pacientes pediátricos y la experiencia es limitada para recomendar su uso en forma rutinaria.

La dosis publicada del lisinopril es $0,07-0,1 \mathrm{mg} / \mathrm{kg} /$ día, en una sola dosis vía oral y la de ramipril $2-6 \mathrm{mg} / \mathrm{m}^{2} /$ día en una sola dosis diaria, vía oral, máxima dosis $10 \mathrm{mg} /$ día $^{4,5}$.

Los dos medicamentos que se emplean rutinariamente en niños son el captopril y el enalapril. Siempre se debe comenzar por las dosis más bajas e incrementar la de manera progresiva. En adultos el mejor efecto se obtiene con las dosis más altas. La desventaja de emplear el captopril es la tos que puede inducir.

Los IECA se deben emplear con precaución en menores de 4 meses de edad por el riesgo de falla renal. En nuestra experiencia evitamos su empleo en lactantes menores.

Los ARAll se han usado cuando hay efectos indeseables secundarios de los IECA, especialmente la tos. No hay un beneficio clínico superior a los IECA.

\section{Betabloqueadores}

El empleo de betabloqueadores no específicos en pacientes pediátricos en falla cardíaca es muy cuestionado.

Los mecanismos que se argumentan para justificar su utilización son los siguientes:

- Evitan la alteración de la matriz extracelular y el desacople de receptores $\beta$ y pueden reversar los efectos de remodelación cardíaca en pacientes en falla ${ }^{24}$.

- Disminuyen la frecuencia cardíaca. En paciente en falla por lo general los estímulos neurohormonales producen taquicardia como mecanismo compensador, pero este mecanismo es deletéreo al no permitir el llenado y la eyección adecuados del volumen sanguíneo del ventrículo izquierdo.

- Disminuyen la producción de radicales libres y la liberación de factores neurohormonales adversos.

- Reducen la presencia de arritmias y la progresión lenta de la falla cardíaca ${ }^{25-29}$.

- Inhiben el gen fetal responsable en parte de la activación de mecanismos apoptóticos.

- Reducen la tensión de la pared ventricular, crucial para que la fibra miocárdica se recupere ${ }^{30}$.

Los mecanismos neurohormonales involucran activación del sistema simpático, sistema, renina-angiotensinaaldosterona, llevando a disfunción miocárdica intrínseca, apoptosis y remodelación.
El meta-análisis de diferentes estudios ha demostrado que el empleo de beta-bloqueadores produjo una disminución del $32 \%$ en el riesgo de muerte, un $41 \%$ reducción en el riesgo de hospitalización por falla cardíaca y un incremento del $29 \%$ en la fracción de eyección en pacientes adultos ${ }^{31}$.

Los estudios hasta la actualidad han demostrado un mejor efecto del carvedilol comparado con el metoprolol 27,28,32-34.

El reporte del único trabajo aleatorizado que se ha publicado no mostró beneficio con el empleo de carvedilol en niños en falla cardíaca ${ }^{35}$. Es pertinente anotar que las dosis en este estudio no fueron las recomendadas actualmente y el intervalo de administración del carvedilol fue cada 12 horas y no cada 8 horas según la indicación más reciente para menores de 5 años. No existen estudios aleatorizados en niños que demuestren el beneficio en pacientes con corazón univentricular de morfología izquierda con falla cardíaca asintomáticos. Hay reportes de pequeñas series de casos que muestran beneficio en pacientes con miocardiopatía dilatada y corazón con ventrículo único de morfología derecha, en quienes hay mejoría de la fracción de eyección y de la clase funcional ${ }^{36}$.

Hay tres medicamentos betabloqueadores que se pueden emplear: metoprolol, carvedilol y bisoprolol; este último no se emplea en menores de $50 \mathrm{~kg}$ de peso.

El carvedilol tiene efectos antioxidantes e inhibe la disfunción miocárdica inducida por liberación de radicales libres. Otro mecanismo de acción del carvedilol está mediado por la estimulación de la liberación de óxido nítrico por el endotelio vascular ${ }^{31,37}$.

En falla cardíaca, los adultos presentan down-regulation y desensibilización de los receptores betaadrenérgicos, secundario a la estimulación crónica de las catecolaminas, que parece explicar el beneficio de los betabloqueadores. En los niños este efecto es diferente; en aquellos con miocardiopatía dilatada tienen una disminución tanto de receptores $\beta 1$ como $\beta 2$, en adultos solo disminuyen los receptores $\beta 1$, los $\beta 2$ se preservan ${ }^{38,39}$.

\section{Análogos del péptido natriurético (Neseritide)}

Constituyen una nueva herramienta para el manejo de la falla cardíaca aguda y crónica, su mecanismo de acción es por su efecto vasodilatador y natriurético, permitiendo reducir la postcarga y la sobrecarga de volumen. Su empleo en pacientes pediátricos es limitado y faltan estudios que demuestren su beneficio sobre otras terapias ${ }^{40,41}$.

\section{Bloqueadores de receptores de la vasopresina}

La vasopresina es un mediador importante en la regulación del tono vascular, y la osmolaridad sérica; es, además, responsable en parte de los cambios en la estructura miocárdica en los pacientes en falla cardíaca.

Nuevos medicamentos antagonistas de la vasopresina (tolvaptan, conivaptam) están en estudios clínicos en fase III y han demostrado un efecto benéfico cuando se emplean en falla cardíaca asociados a diuréticos ${ }^{4,42}$. No hay estudios que demuestren un incremento en la sobrevida con su empleo. No hay datos para recomendar su uso en niños. 


\section{Soporte mecánico}

Existen diferentes tipos de terapias no farmacológicas que pueden ser empleadas en niños para el tratamiento de cuadro de shock cardiogénico que permiten el reposo miocárdico y disminuir al mínimo el consumo de oxígeno miocárdico en pacientes con patologías reversibles ${ }^{43}$.

Tales terapias son los sistemas de soporte cardíaco extracorpóreos y los sistemas de asistencia ventricular (VAD) que pueden ser uni o biventriculares, y la oxigenación por membrana extracorpórea (ECMO), la cual se utiliza cuando hay compromiso pulmonar importante asociado a falla cardíaca.

Las indicaciones más frecuentes para estos tipos de soporte son miocarditis aguda ${ }^{44,45}$, síndrome de bajo gasto postoperatorio, puente para trasplante cardíaco, hipertensión pulmonar severa o como terapia de destino.

Hay varios sistemas disponibles, los cuales pueden ser extracorpóreos o paracorpóreos. El único sistema extracorpóreo que se puede emplear por largos períodos de tiempo es el Berlin Heart ${ }^{\oplus}$; se puede implantar desde la etapa neonatal, como puente para trasplante. El paciente debe permanecer hospitalizado todo el tiempo. Los otros sistemas extracorpóreos (Cardiohelp ${ }^{\oplus}$, Centrimag ${ }^{\oplus}$ ) están diseñados para períodos cortos de soporte.

Los sistemas paracorpóreos se pueden usar en niños grandes con superficie corporal alrededor de $1 \mathrm{~m}^{2}$. En la actualidad se dispone de dos sistemas (el HeartMate II ${ }^{\oplus}$ III, y el HeartWare $\left.{ }^{\oplus}\right)$. La experiencia pediátrica es mayor con el empleo del HeartWare ${ }^{\circledR}$. Su aprobación por la FDA (U. S. Food and Drug Administration) es como puente a trasplante en la edad pediátrica, pero se han empleado en algunos centros como terapia de destino ${ }^{46}$.

Hay sistemas de soporte para períodos cortos de tiempo como el Impella ${ }^{\oplus}$, que se emplea para niños grandes y se implanta en la sala de Hemodinamia en la raíz de la aorta impulsando la sangre eyectada del ventrículo izquierdo permitiendo cierto grado de reposo miocárdico.

Ninguna de estas técnicas está exenta de complicaciones; entre ellas las más frecuentes son secundarias a la anticoagulación sistémica, como hemorragias intracraneanas, y en segundo lugar están las infecciones.

El empleo del soporte vital extracorpóreo ha disminuido la mortalidad en forma importante en el shock cardiogénico por miocarditis.

El empleo de sistemas implantables de asistencia extracorpórea en niños como terapia de destino, está limitado por el tamaño de los dispositivos ${ }^{47}$.

\section{Terapia génica}

Uno de los fenómenos que se estudian en la actualidad como tratamiento de la falla cardíaca, es la capacidad que tiene el músculo cardíaco para regenerarse y formar nuevamente miocitos funcionales.

La terapia génica vía manipulación del ADN, ARN o ambos y su expresión es muy atractiva en el tratamiento de miocardiopatías de herencia genética y miocardiopatías virales.

La forma clásica de la terapia génica es la inserción de ADN dentro del núcleo celular. Este ADN es luego transcrito a un ARNm para producir una proteína terapéutica ${ }^{48}$.
Para la terapia génica que requiere transcripción e implementar su efecto existen barreras adicionales, que incluyen el transporte ineficiente del vector con el material terapéutico al núcleo, transcripción ineficiente de los genes terapéuticos e inactivación de los genes terapéuticos por las células blanco ${ }^{49}$.

Aunque el ingreso a la célula de la información genética puede demostrarse después de la administración de $A D N$ o ARN denudado, la eficacia de esta transferencia usualmente es baja.

El ADN o ARN que se pretende emplear para terapia génica es típicamente administrado a través de un vector que facilite la entrada de los ácidos nucleicos al núcleo celular. Pueden emplearse vectores virales o no-virales. Los virus están compuestos por una capa proteica exterior llamada cápside y una carga genética interior. La cápside proteica viral tiene un sistema complejo que incrementa la adherencia celular, así como la internalización y entrega de los ácidos nucleicos virales ${ }^{50}$. El problema encontrado con estas terapias es el riesgo de formación de lesiones tumorales.

\section{Células madre}

Existe un nuevo campo de investigación para la siembra de células madre en sitios dañados del músculo cardíaco y generar nuevos miocitos que reemplacen el tejido muerto ${ }^{36,51}$.

Las células cardíacas progenitoras (CCP) pueden multiplicarse in vitro y transformarse en cardiomiocitos para luego administrarse por vía intracoronaria para que pueblen las zonas lesionadas. Esta terapia tiene gran potencial para el tratamiento de la falla cardíaca en Pediatría; el gran número de CCP en recién nacidos y lactantes menores abre la posibilidad de esta terapia a un gran número de pacientes. Otro gran progreso ha sido la tecnología para inducir la formación de células madre pluripotenciales (CMP); se reprograman células endoteliales diferenciadas a células indiferenciadas en un estado casi-embriónico y luego a cardiomiocitos. Cuando las CMP se diferencian hacia cardiomiocitos son una fuente potencial de tejido autólogo para la terapia celular ${ }^{52}$. En la actualidad se está trabajando en la diferenciación de células epiteliales maduras del paciente en cardiomiocitos, sin pasar por la fase de células madre pluripotenciales.

Hay un reporte de caso del empleo exitoso de células madres pluripotenciales en un niño en postoperatorio tardío de Norwood, con mejoría de la función del ventrículo único y de la insuficiencia tricuspídea ${ }^{53}$.

Los resultados de las investigaciones sugieren que hay muchas otras variaciones genéticas que contribuyen a la variabilidad en la respuesta a los tratamientos de la falla cardíaca, incluyendo polimorfismo en los genes que codifican la enzima convertidora de la angiotensina y la sintetasa de aldosterona ${ }^{54}$.

\section{Terapia de resincronización}

Ha mostrado utilidad en la falla cardíaca de pacientes adultos refractarios al tratamiento médico y que cumplan los siguientes criterios: fracción de eyección < $35 \%$, clase funcional de la NYHA III-IV, QRS > de $120 \mathrm{~ms}$. Los hallazgos de los estudios en adultos han impulsado su empleo en la población pediátrica. En este grupo los resultados han sido muy 
variables y dependerán de la patología de base. También hay preocupación debido al poco tiempo de seguimiento en los trabajos reportados y no hay información de qué sucederá con estos pacientes a largo plazo. Aunque algunos estudios han demostrado un efecto benéfico en pacientes pediátricos con cardiopatías congénitas, hay algunos aspectos que llaman la atención; uno de ellos es que la población estudiada es altamente heterogénea con diferentes sustratos anatómicos (ventrículo izquierdo sistémico, ventrículo derecho sistémico, ventrículo único), además de diferentes etiologías de la falla cardíaca. Los reportes muestran mejoría en la clase funcional o fracción de eyección desde un 29 a un 74\% de los pacientes; así mismo, todos referencian una disminución del QRS a menos de 120 ms. También es anotar que en los estudios publicados la mayoría de los pacientes estaban previamente en clase funcional I-II, lo cual indica falla cardíaca leve a moderada. En los pocos estudios actuales, los mejores resultados se han visto cuando se trata de un corazón biventricular y que haya tenido un marcapasos implantado previamente, y de morfología univentricular en falla cardíaca. Se han encontrado los mejores resultados cuando el ventrículo único es de morfología izquierda ${ }^{55-57}$.

La terapia de resincronización cardíaca es una más de la terapias que pueden utilizarse en un grupo pequeño de pacientes pediátricos en falla cardíaca refractaria al tratamiento médico antes de considerar el trasplante cardíaco o un sistema de asistencia ventricular ${ }^{58}$.

La terapia de resincronización no se recomienda en la guías internacionales actuales; se requieren estudios aleatorizados antes de utilizar la resincronización en la práctica clínica en este subgrupo de pacientes con cardiopatías congénitas $^{59}$.

\section{Trasplante cardíaco}

El pronóstico de la miocardiopatía dilatada como causa de falla cardíaca en niños aún es pobre. Después de la hospitalización inicial el riesgo de falla cardíaca, muerte, trasplante o rehospitalización durante el primer año es del $79 \%{ }^{55,60}$, en tanto que la sobrevida sin trasplante a los 5 años es del $51 \%{ }^{56,61}$.

En niños los resultados del manejo médico no son tan prometedores como en adultos, de ahí que un número relativamente mayor de niños en falla cardíaca requerirá trasplante cardíaco en los primeros años de vida. La sobrevida actuarial de los trasplantes pediátricos en general es buena, pero dependen de la edad en la cual se realizó el trasplante; cuanto más pequeño mayor la sobrevida del injerto. En los trasplantes realizados en menores de 1 año de edad existe menor riesgo de rechazo comparado con los realizados en adolescentes ${ }^{62,63}$. La sobrevida del injerto para los trasplantados en la edad neonatal es de 19,2 años, en la edad escolar de 15,6 años y en la adolescencia de 11,9 años. Hay un número creciente de pacientes que fueron sometidos a cirugías paliativas definitivas por cardiopatías congénitas de corazón univentricular. Este grupo de pacientes tiene mayor riesgo de mortalidad en comparación con aquellos con corazón biventricular.

La mayor mortalidad se encuentra en el primer año de vida. Después de un trasplante exitoso, el riesgo de mortalidad se asocia primordialmente a vasculopatía coronaria del corazón trasplantado, enfermedad linfoproliferativa post-trasplante y falta de adherencia al régimen de medicamentos ${ }^{59,64}$.

Hay factores que están asociados a mayor mortalidad intrahospitalaria en el paciente trasplantado, como alteraciones de las pruebas de función renal y elevación de creatinina, empleo de ventilación mecánica, asistencia ventricular o ECMO. Un paciente con alteración significativa de las pruebas anteriormente mencionadas, que requiere ECMO, tiene riesgo de mortalidad intrahospitalaria del $69 \%{ }^{60,65}$.

\section{Pronóstico}

La mortalidad de los pacientes con falla cardíaca crónica es elevada, particularmente durante los primeros seis meses, con un descenso progresivo de la supervivencia durante los primeros cuatro años. Entre el quinto al octavo año la mortalidad se estabiliza y después de los ocho años presenta otro pico de mortalidad. A los cinco años la mortalidad puede llegar a un $60 \%{ }^{66}$.

\section{Conclusiones}

La falla cardíaca en Pediatría es una entidad poco frecuente pero de alta mortalidad. Después de las cardiopatías congénitas, la causa más frecuente de falla es la miocarditis. La activación del sistema neurohormonal y los mecanismos compensadores son los responsables directos de la presentación clínica, los hallazgos paraclínicos y la perpetuación de la falla cardíaca.

La apoptosis se relaciona de manera directa con el empeoramiento del cuadro clínico en los casos crónicos. El tratamiento actual de la falla está dirigido a regular los mecanismos neurohormonales y a disminuir la tensión de la pared miocárdica, la liberación de catecolaminas endógenas y la apoptosis.

Hay nuevas terapias génicas y de células madre prometedoras a un futuro.

El trasplante cardíaco tiene la limitación de la falta de órganos. El implante de un sistema de asistencia ventricular en forma definitiva puede ser una solución para pacientes adolescentes, pero los sistemas para pacientes pequeños aún están en desarrollo.

\section{Conflicto de intereses}

Ninguno.

\section{Bibliografía}

1. Kantor PF, Mertens LL. Heart failure in children Part I: clinical evaluation and initial medical management. Eur J Pediatr. 2010;169:269-79.

2. Lother A, Hein L. Pharmacology of Heart failure: From basic science to novel therapies. Pharmacol Ther. 2016;166:136-49.

3. Kirk R, Dipchand Al, Rosenthal DN, Addonizio L, Burch M, Chrisant M, et al. The International Society for Heart and Lung Transplantation Guidelines for the management of pediatric Heart failure: Executive summary. J Heart Lung Transplant. 2014;33:890-908.

4. Kantor PF, Lougheed J, Dancea A, McGuillon M, Barbosa N, Chan $C$, et al. Society Guidelines. Presentation, Diagnosis, 
and Medical Management of Heart Failure in Children: Canadian Cardiovascular Society Guidelines. Canadien J Cardiol 2013;29:1535-1552.

5. Timberlake K, Kantor PF. Pharmacologic therapy of heart failure in children. Part of a special series on Paediatric Pharmacology, guest edited by Gianvincenzo Zuccotti, Emilio Clementi, and Massimo Molten. Pharmacological Res. 2011;64:427-30.

6. Kay JD, Colen SD, Graham TP Jr. Congestive herat failure in pediatric paients. Am Heart J. 2001;142:923-92.

7. Shaddy R. Optimizing treatment for chronic congestive heart failure in children Crit Care med. 2001;29:S237-40.

8. Eshaghian S, Horwich TB, Fonarow GC. Dose-dependent association between use of loop diuretics and mortality in advanced systolic heart failure. Am J Cardiol. 2006;98:1416-7.

9. Alon US, Scagliotti D, Garola RE. Nephrocalcinosis and nephrolithiasis in infants with congestive heart failure treated with furosemida. J Pediatr. 1994;125:149-51.

10. Felker GM, O’Connor CM. Inotropic therapy for heart failure: An evidence-based approach. A Heart J. 2001;142:393-401.

11. Ratnapalan SR, Griffiths K, Moldovan-Costei A, Benson L, Koren G. Digoxin-carvedilol interactions in children. J Pediatr. 2003;142:572-4.

12. Digitalis investigators group. The effect of digoxin on mortality and morbidity in patients with heart failure. N Eng J Med. 1997;336:525-33.

13. Hummel J, Rücker G, Stiller B. Prophylactic levosimendan for the prevention of low cardiac output syndrome and mortality in paediatric patients undergoing surgery for congenital heart disease (Review)) Cochrane Database of Systematic Reviews 2017, Issue 8. Art. No.: CD011312. DOI: 10.1002/14651858.CD011312.pub3.

14. Rossano JW, Cabrera AG, Jefferies JL, Naim MPH, Maryam Y, Humlicek T. Pediatric Cardiac Intensive Care Society 2014 Consensus Statement: Pharmacotherapies in Cardiac Critical Care Chronic Heart Failure. Pediatr Crit Care Med. 2016;17:S20-34.

15. Hasenfuss G, Teerlink JR. Cardiac Inotropes: Current agents and future directions. Eur Heart J. 2011;32:1838-45.

16. Captopril-Digoxin Multicenter Research Group. Comparative effects of therapy with captopril and digoxin in patients with mild to moderate heart failure The Captopril Multicenter Research Group. JAMA. 1988;259:539-44.

17. Cohn JM, Johnson G, Ziesche S, Cobb F, Francis G, Tristani F, et al. A comparison of enalapril with hydralazine-isosorbide dinitrate in the treatment of chronic congestive heart failure. N Eng J Med. 1991;325:303-10.

18. SOLVD investigators. Effect of enalapril on survival in patients with reduce left ventricular ejection fractions and congestive Heart failure. N Eng J Med. 1991;325:293-302.

19. Lewis $A B$, Chabot $M$. The effect of treatment with angiotensine converting enzyme inhibitors on survival of pediatric patients with dilated cardiomyopathy. Pediatr Cardiol. 1993;14:9-12.

20. ATLAS study groupPacker M, Poole-Wilson PA, Armstrong PW. Comparative effects of low and high dose of the angiotensina converting enzyme inhibitor lisinopril, on morbidity and mortality in chronic heart failure. Circulation. 1999;100:2312-8.

21. Van Veldhuissen DJ, Genth-Zotz S, Brouwer J. High-versus low dose ACE inhibition in chronic heart failure. A doubleblind placebo controlled study of imidapril. J Am Coll Cardiol. 1998;32:1811-8.

22. Montigny M, Davidson A, Fouron JC. Captopril in infants for congestive heart failure secondary to a large ventricular leftto-right shunt. Am J Cardiol. 1989;63:631-3.

23. Kyong-J L, Shi-Joon Y, Holby H, et al. Acute effects of the ACE inhibitor enalaprilat on the pulmonary, cerebral and systemic blood flow and resistance after the bidirectional cavopulmonary connection. Heart. 201;97(16):1343-48.
24. Metra M, Nodari S, Parrinello G, Glubbini R, Manca C, Del Cas L. Marked improvement in left ventricular ejection fraction during long term blockade in patients with chronic heart failure: clinical correlates and prognostic significance. Am Heart J. 2003;145:292-9.

25. Bruns LA, Kichuk Chrisant M, Lamour JM, Shaddy R, Pahl E, Blume ED, et al. Carvedilol therapy in pediatric heart failure: An Initial multicenter experience. J Pediatr. 2001;138:505-11.

26. Spicer RL. Carvedilol- a new dimension in pediatric heart failure therapy. J Pediatr. 2001;138:457-8.

27. Shaddy RE, Curtin EL, Sower B, Tani LY, Burr J, LaSalle B, et al. The pediatric randomized trial in children with chronic heart failure: Rationale and design. Am Heart J. 2002;144:383-9.

28. Bollano E, Tang MS, Hjalmarson A, Waagstein F, Andersson B. Different response to dobutamine in the presence of carvedilol or metoprolol in patients with chronic heart failure. Heart. 2003;89:621-4.

29. Azeka E, Franchini-Ramires JA, Bocchi EA. Deslisting of infants and children From the heart transplantation waiting list after carvedilol treatment. J Am Coll Cardiol. 2002;40:2034-8.

30. Kalinowski L, Dobrucki LW, Szczepanska-Konel M, Jankowski M, Martyniec L, Angielski S, Malinski T. Third-generation b blockers stimulate Nitric Oxide release from endothelial cells through ATP efflux. A novel antihypertensive action. Circulation. 2003;107:2747-52.

31. Lechat P, Packer M, Chalon S, Cucherat M, Arab T, Boissel JP. Clinical effects of beta-Adrenergic blockade in chronic heart failure: A metaanalysis of double-blind, placebo-controlled, randomized trials. Circulation. 1998;98:1184-91.

32. Towbin JA, Bowles NE. The failing heart. Nature. 2002;415:227-33.

33. Poole-Wilson PA, Swedberg K, Cleland JGF, Di Lenarda A, Hanrath P, Komajda M, et al. Comparison of carvedilol and metoprolol on clinical outcomes in patients with chronic heart failure in the Carvedilol or Metoprolol European Trial (COMET): randomised trial. Lancet. 2003;362:7-13.

34. Cleland JGH, Swedberg K, Poole-Wilson PA. Successes and failures of current treatment of heart failure. Lancet. 1998;352 suppl:1928-48.

35. Bolli R. Regeneration of the human heart- No chimera? N Eng J Med. 2002;346:55-6.

36. Huang $M$, Zhang $X$, Chen $S$, Sun $Y$, Xiao $Y$, Sun J, et al. The effect of carvedilol treatment on chronic heart failure in pediatric patients with dilated cardiomyopathy: a prospective, randomized-controlled study. Pediatr Cardiol. 2013;34:680-5.

37. Laer S, Behn F, Eiselt M, Scholz H, Venzke A, Meibom B, et al. Carvedilol therapy in pediatric patients with congestive Heart failure: A study investigating clinical and pharmacokinetic parameter. Am Heart J. 2002;143:916-22.

38. Miyamoto SD, Stauffer BL, Nakano S, Sobus R, Nunley K, Nelson P, et al. Beta Adrenergic adaptation in paediatric idiopathic dilated cardiomyopathy. Eur Heart J. 2014;35:33-41. https://doi.org/10.1093/eurheartj/ehs229

39. Debra A. Dodd. Pediatric heart failure and transplantation: where are we in 2013. Curr Opin Pediatr. 2013;25:553-60.

40. Mahle, Wt, Cuadrado AR, Kirshbom PM, Kanter KR, Simsic JM. Neseritide in infants and children with congestive heart failure. Pediatr Crit Care Med. 2005;6:543-6.

41. Simsic JM, Scheurer M, Tobias JD, Berkenbosh J, Schechter W, Madera F, et al. Perioperative effects and safety of Neseritide following cardiac surgery in children. J Intensive Care Med. 2006;21:22-6.

42. Lee CR, Watkins ML, Patterson JH, Gattis W, OĆonnor CM, Gheorghiade $M$, et al. Vasopress new target for the treatment of heart A. Am Heart failure.J. 2003;146:9-18.

43. Shekerdemian L. Nonpharmacologic treatment of acute heart failure. Curr Opin Pediatr. 2001;13:240-6. 
44. Rajagopal SK, Almond CS, Laussen PC, Rycus PT, Wypij D, Thiagarajan RR. Extracorporeal membrane oxygenation for the support of infants, children, and young adults with acute myocarditis: A review of the Extracorporeal Life Support Organization registry*. Crit Care Med. 2010;38:382-7.

45. Kuan-Miao L, Ming-Hsiu L, Kai-Sheng H, Hsuan-Chang K, MingChou C, Jiunn-Jye $S$, et al. Impact of extracorporeal membrane oxygenation on acute fulminant myocarditis-related hemodynamic compromise arrhythmia in children. Pediatrics and Neonatology. 2016, https://doi.org/10.1016/j.pedneo.2016.02. 002 .

46. Kirklin JK. Advances in mechanical assist devices and artificial hearts for children. Curr Opin Pediatr. 2015;27:597-603.

47. Gandhi SK, Epstein DJ. Ventricular assist device support in children. In: Wheeler DS, Wong H, Chanley T. (eds.). Pediatric critical care medicine. Vol. 2. London: Springer-Verlag; 2014.

48. Penny DJ, Vick WG III. Novel therapies in childhood heart failure: today and tomorrow. Heart Failure Clin. 2010;6:591-621.

49. Vaughan EE, DeGiulio JV, Dean DA. Intracellular trafficking of plasmids for gene therapy: mechanisms of cytoplasmic movement and nuclear import. Current Gene Therapy. 2006;6:671-81

50. Lyon AR, Sato M, Hajjar RJ, Samulski J, Harding SE. Gene therapy: targeting the myocardium. Heart. 2008;94:89-99.

51. Quaini F, Urbanek K, Beltrami AP, Finato N, Beltrami CA, NadalGuinard B, et al. Chimerism of the transplanted heart. N Eng J Med. 2002;346:5-15.

52. Files MD, Boucek RJ. 'Shovel-Ready' applications of stem cell advances for pediatric heart Disease. Curr Opin Pediatr. 2012;24:577-83.

53. Rupp S, Zeiher AM, Dimmeler S, Tonn T, Bauer J, Jux C, et al. A regenerative strategy for heart failure in hypoplastic left heart syndrome: intracoronary administration of autologous bone marrow-derived progenitor cells. J Heart Lung Transplant. 2010;29:574-7.

54. Penny DJ, Wesley Vick G. Novel therapies in childhood heart failure: Today and tomorrow. Heart Failure Clin. 2010;6:591-621.

55. Cecchin F, Frangini PA, Brown DW, Fynn-Thompson F, Alexander ME, Triedman JK, et al. Cardiac resynchronization. therapy (and multisite pacing) in pediatrics and congenital heart disease: five years experience in a single institution. J Cardiovasc Electrophysiol. 2009;20:58-65.

56. Van der Hulst AE, Delgado V, Blom NA, van de Veire NR, Schalij MJ, Jeroen JB, et al. Cardiac resynchronization therapy in paediatric and congenital heart disease patients. Eur Heart J. 2011;32:2236-46.

57. Dubin AM, Janousek J, Rhee E, Strieper MJ, Cecchin F, Law IH, et al. Resynchronization therapy in pediatric and congenital heart disease patients: an international multicenter study. J Am Coll Cardiol. 2005;46: 2277-83.

58. Janousek J, Gebauer RA, Abdul-Khaliq H, Turner M, Kornyei L, Grollmuß O, et al. Cardiac resynchronization therapy in paediatric and congenital heart disease: differential effects in various anatomical and functional substrates. Heart. 2009;95:1165-71.

59. Thamboa JB, Dos Santos P, Bordacharb P. Cardiac resynchronization therapy in patients with congenital heart disease Resynchronisation cardiaque et cardiopathies congénitales. Arch Cardiovasc Dis. 2011;104:410-6.

60. Hollander SA, Bernstein D, Yeh J, Dao D, Sun HY, Rosenthal D. Outcomes of children following a first hospitalization for dilated cardiomyopathy. Circ Heart Fail. 2012;5:437-43.

61. Alvarez JA, Orav EJ, Wilkinson JD, Fleming LE, Lee DJ, Sleeper $\mathrm{LA}$, et al. Competing risks for death and cardiac transplantation in children with dilated cardiomyopathy: results from the Pediatric Cardiomyopathy Registry. Circulation. 2011;124:814-23.

62. Boucek RJ, Boucek MM. Pediatric heart transplantation. Curr Opin Pediatr. 2002;14:611-9.

63. Hosepoud JD, Bennett LE, Kerk BM, Boucek MM, Novick RJ. The registry of the International Society for Heart and Lung Transplantation: 18th official report 2001. J Heart Lung Transplant. 2001;20:805-15.

64. Dodd DA. Pediatric heart failure and transplantation: where are we in 2013? Curr Opin Pediatr. 2013;25:553-60.

65. Almond CS, Gauvreau K, Canter CE, Rajagopal SK, Piercey GE, Singh TP. A risk-prediction model for inhospital mortality after heart transplantation in US children. Am J Transplant. 2012;12:1240-8.

66. Ross RD, Bolling RO, Pinsky WW. Grading the severity of congestive heart failure in infants. Pediatr Cardiol. 1992;13:72-5. 